implications - societal, ethical, personal. By reflecting the work from new angles - and being confronted with a different way of thinking - new insights emerge for both the participating scientists and artists.

Scientific research does not exist in a bubble, but is relevant in its societal context. It should be motivating that people outside of academia increasingly engage with scientific research as citizen scientists, DIY biologists or artist-researchers. To engage in art-science programmes is an opportunity for research institutes to further open up and proactively engage in a cultural discourse, as well as for scientists to negotiate their work with the public. Especially for young researchers, this can be a very meaningful experience, much like a field experiment to explore the broader implications of their research and gain new insights.

It is the role of curators to foster such opportunities and try to make sure they are fruitful for both sides - while contributing to an inclusive and meaningful dialogue around the relevance and implications of science in society.

\section{Christian Rauch \\ STATE Studio, Berlin, Germany. \\ e-mail:cr@state-studio.com}

Published online: 7 May 2019

https://doi.org/10.1038/s41565-019-0449-3

\title{
Questioning life
}

Art-based absurd scenarios serve as a vector to challenge perceptions of emergent biotechnologies.

believe art has the potential to initiate public debate and critical reflection on a unique cultural moment in which we are witnessing the unprecedented evolution of biotechnologies and various modes of liminal lives that defy traditional understandings of life. I create artworks designed to raise questions about the influence of current and emergent biotechnologies on the shifting forces that govern and determine life, death and sentience.

Since 2001, biology laboratories have been my art studio. It is there that my creative process takes place; tissue engineering, electrophysiology and other biological techniques are my artistic media.

My research (http://guybenary.com/) explores a number of fundamental themes, namely life and death, cybernetics and artificial life. I consciously approach processes capable of transforming bodies or living biological materials from artistic, philosophical and ethical perspectives, and I make use of new cybernetic technologies to create artworks that re-evaluate our understanding of life and the human body. I use biotechnologies in a subversive way, attempting to problematize them by putting forward absurd scenarios. Visual strategies are used to help lure viewers into exploring the artworks in a manner that sparks a dialogue about the future of these technologies and encourages them to re-evaluate their own perceptions and beliefs.

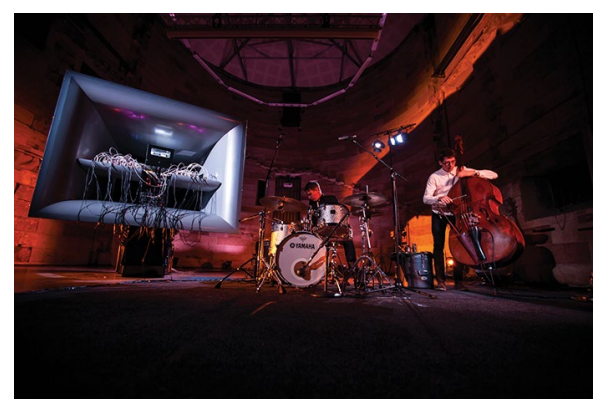

Musicians improvising with cellF in a live performance. Credit: Alex Davies

For example, cellF is a collaborative project (with artists Nathan Thompson, Andrew Fitch and Darren Moore, and scientists Stuart Hodgetts, Mike Edel and Douglas Bakkum) that deals with the embodiment of neural networks. It is my self-portrait but also the world's first living neural synthesizer. cellF's 'brain' is made of my own neurons that grow on a multi-electrode array interface, and it controls analogue synthesizers in real time. It is a completely autonomous analogue instrument. Musicians are invited to play with cellF in special one-off shows. The human-made music is fed to the neurons as stimulation, and the neurons 'respond' by controlling the synthesizers. Together they perform live, reflexive post-human sound pieces.

Another example is Bricolage (developed with Nathan Thompson and
Sebastian Diecke). Here, the focus is not neuronal data but the liveliness of the biological matter through physical movement and the behaviour of cardiomyocytes. In Bricolage we create artistic, autonomous, biological robots that have the ability to self-assemble in the gallery space during the exhibition. These living kinetic sculptures are derived from sources loaded with the engrained cultural associations of three main components: blood, heart and silk. Bricolage develops these associations and explores their cultural meanings, while at the same time manifesting the visceral behaviour of the cardiomyocytes through their movement.

My work addresses a 'new materialist' question, underpinned by the belief that artistic practice can act as a vector for thought. What is the potential for artworks using biological and/or robotic technologies to evoke responses and to shift perceptions surrounding the understandings of life, death, sentience and the materiality of the human body?

\section{Guy Ben-Ary}

SymbioticA, Centre of Excellence for Biological Arts, School of Human Sciences, University of Western Australia, Perth, Western Australia, Australia. e-mail: guy.benary@uwa.edu.au

Published online: 7 May 2019 https://doi.org/10.1038/s41565-019-0444-8 